\title{
Neural derivation of human dental pulp stem cells via neurosphere technique
}

\author{
Bojnordi $\mathrm{MN}^{1}$, Haratizadeh $\mathrm{S}^{1}$, Darabi $\mathrm{S}^{2}$, Hamidabadi $\mathrm{HG}^{1}$ \\ Immunogenetic Research Center, Department of Anatomy \& Cell Biology, Faculty of Medicine, \\ Mazandaran University of Medical Sciences, Sari, Iran. h.ghasemi@mazums.ac.ir
}

\begin{abstract}
INTRODUCTION: Human dental pulp stem cells (hDPSCs) are multipotent stem cells providing an autologous noninvasive cell source. The study evaluates the neurogenic potential of hDPSCs using neural growth factor inducers and neurosphere technique.

METHODS: The hDPSCs were differentiated into neurons using neural induction medium containing retinoic acid (RA). Neuroprogenitor cells were evaluated for nestin and NF68 using immunocytochemistry. The mature neuron markers, MAP-2 and $\beta$-tubulin, were investigated at the end stage of induction phase.

RESULTS:The neuroprogenitor differentiation was confirmed by immunostaining for nestin and NF68 markers. The differentiated neurons were positive for specific neuron markers, namely for MAP-2 and $\beta$-tubulin. The results indicated that the neural differentiation medium and neurosphere technique improve the generation of neuroprogenitor cells as well as mature neurons via exhibiting specific neural markers, namely nestin, NF68, MAP-2 and $\beta$-tubulin.

CONCLUSION: Our findings highlight the differentiation capacity of hDPSCs via neurosphere technique in the presence of neural inducers for mesenchymal stem cells. It is suggested that the neural differentiation potential of hDPSCs can be exploited as a source of stem cells for therapy of neurodegenerative diseases (Fig. 5, Ref. 20). Text in PDF www.elis.sk.

KEY WORDS: mesenchymal stem cells, dental pulp stem cells, neuron, neuroprogenitor cells, multipotent stem cells.
\end{abstract}

\section{Introduction}

Human dental pulp stem cells are multipotent stem cells possibly considered to be a new noninvasive autologous source for MSCs (Gronthos et al, 2000; Sloan et al, 2007). Their origin is similar to that of neural cells. Therefore their neural differentiation capacity can be acceptably exhibited (Huang, 2008; Gronthos et al, 2000).

The hDPSCs can differentiate into various lineages such as adipocytes, osteocytes, neurons and glial cells (Sloan, 2007; Gronthos $\mathrm{S}$ et al, 2002). Although the ability of hDPSCs to differentiate into neurons has already been shown, the maturity has not been yet investigated. The aim of this research was to improve the induction technique for in vitro transdifferentiation of hDPSCs into mature neurons in the presence of neural inducers.

${ }^{1}$ Immunogenetic Research Center, Department of Anatomy \& Cell Biology, Faculty of Medicine, Mazandaran University of Medical Sciences, Sari, Iran, and ${ }^{2}$ Department of Anatomy, Faculty of Medicine, Ghazvin University of Medical Sciences, Ghazvin, Iran

Address for correspondence: H.G. Hamidabadi, Immunogenetic Research Center, Department of Anatomy \& Cell Biology, Faculty of Medicine, Mazandaran University of Medical Sciences, Sari, P.O.Box: 4847191971, Iran.

Acknowledgments: This research was funded by a grant from Mazandaran University of Medical Sciences, Sari, Iran (grant No. 1394-140) and Iranian council of stem cell technology.
The derivation of neuroprogenitor cells was evaluated using the expression of specific neuroprogenitor markers, namely nestin and NF68 as.

The differentiation of hDPSCs has been done previously with neural inducers, but there are no results of it being done via the neurosphere technique. Hence, the goal of our research was to improve the neural induction technique for deriving mature neurons from hDPSCs using neural growth factor inducers and neurosphere technique.

\section{Materials and methods}

\section{Culture of hDPSCs}

The hDPSCs were isolated from human dental pulp collected from third molar teeth at the dental clinic of Mazandaran University of Medical Sciences. The tissues were digested mechanically and enzymatically and then they were centrifuged. Then, the supernatant was removed and cultured in DMEM/F12 containing $15 \%$ FBS (Gronthos S et al, 2002).

\section{Multipotency of hDPSCs}

Osteogenic and adipogenic differentiation of hDPSCs was evaluated via oil red $\mathrm{O}$ and alizarin red staining.

Flow cytometry

Isolated cells were examined immunophenotypically for the 

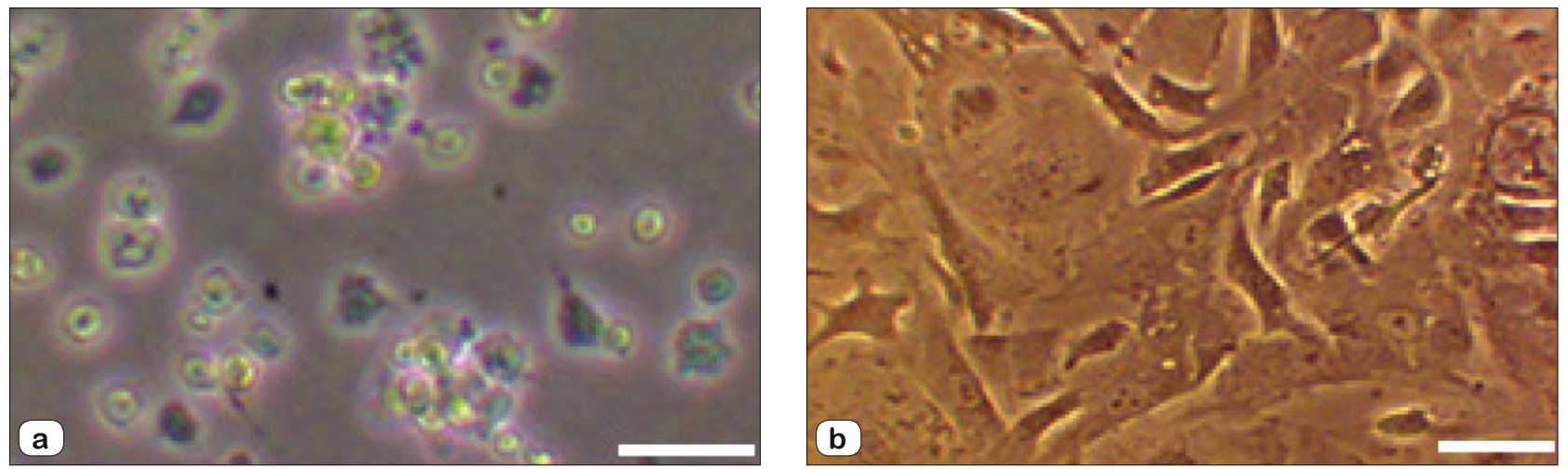

Fig. 1. Phase contrast photography of human dental pulp stem cells after four hours in culture (a). Fibroblastic and spindle morphology of human dental pulp stem cells after fourth passage. Scale bars: $10 \mu \mathrm{m}$.
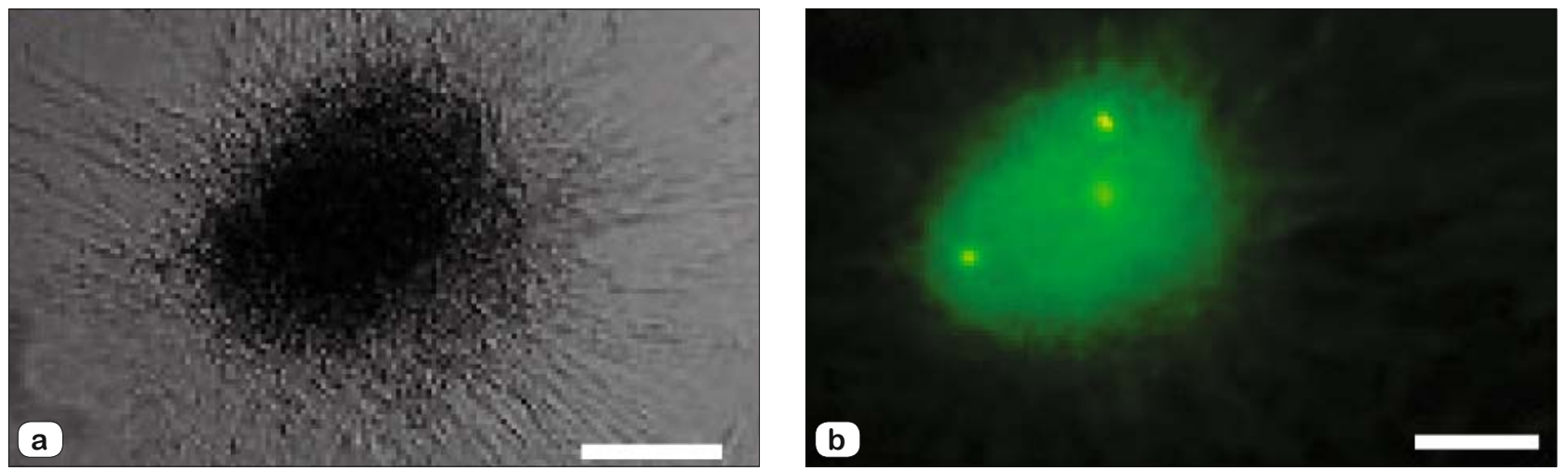

Fig. 2. Phase contrast picture of neurosphere derived from human dental pulp stem cells (a). Neurosphere cells were immunopositive. Scale bars: $\mathbf{5 0 0} \boldsymbol{\mu m}$.
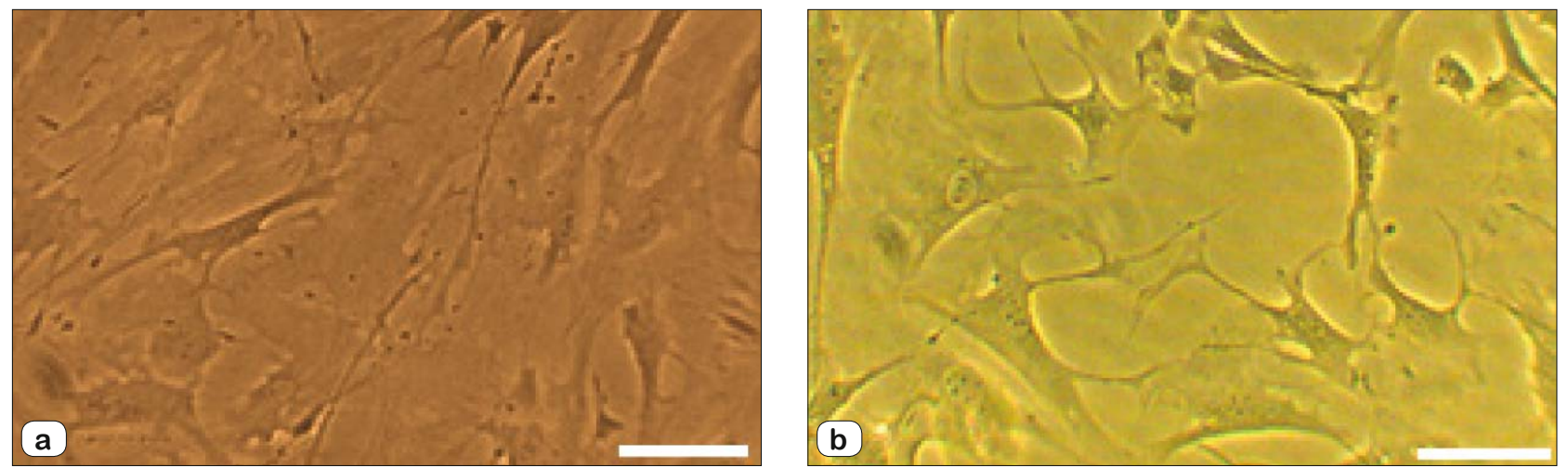

Fig. 3. Neural differentiation of human dental pulp stem cells. Neuroprogenitor cells phenotype (a). Neuron generation at the end stage of induction period (c). Scale bars: $10 \mu \mathrm{m}$.

expression of mesenchymal stem cell markers, namely that of CD44, CD90, CD105.

\section{Induction of neurospheres from hDPSCs}

Neurospheres were generated from hDPSCs according to the previous method. The cells cultured in four passages, were then trypsinized and plated in non-adherence flasks containing neural stem cells culture medium supplemented with $10 \mathrm{ng} / \mathrm{mL}$ of basic fibroblast growth factor, $20 \mathrm{ng} / \mathrm{mL}$ of epidermal growth factor, and B27. Neurospheres were formed in the culture after 7-10 days (Fu et al, 2008).

\section{Differentiation of hDPSCs-derived NSCs into mature neurons}

Neurospheres were differentiated into neurons according to previous research (Darabi et al, 2015). Neural stem cells were plated onto poly-L-lysine-coated coverslips and in order to enhance their terminal neural differentiation they were cultured for 8-10 days in neurobasal medium containing N2 and B27 supplements. 

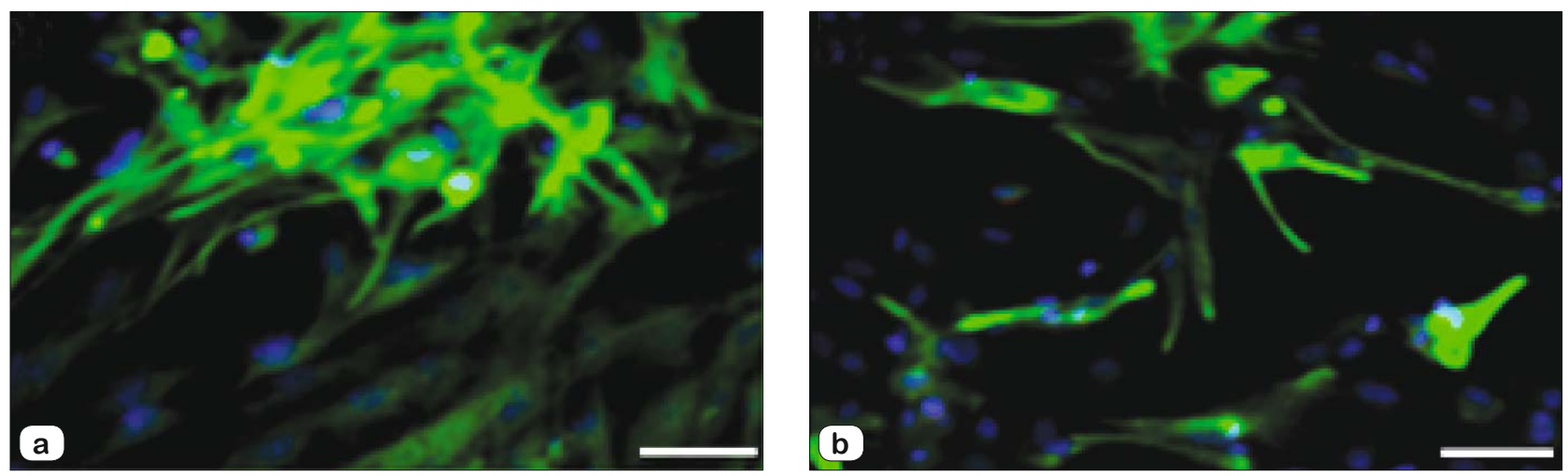

Fig. 4. Immunocytochemistry of differentiated neural stem cells derived from human dental pulp stem cells into neurons. Differentiated cells expressed MAP-2 (a) and tubulin- $\beta$ (b) proteins. Scale bars: $10 \mu \mathrm{m}$.

\section{MTT assay}

Methyl thiazolyl tetrazolium (MTT) was used for evaluating the hDPSCs viability.

\section{Immunofluorescent staining}

The differentiated cells at the end stage of induction phase were investigated for the expression of neuroepithelial specific markers, namely that of nestin and NF68. Mature neural markers, namely MAP-2 and $\beta$-tubulin, were evaluated at the end stage of induction phase. Briefly, the cells were fixed in $4 \%$ paraformaldehyde $(\mathrm{pH}$ 7.4) for half an hour at room temperature. Then they were permeabilized with $0.2 \%$ Triton X-100 for 10 min. After washing with PBS they were blocked with $10 \%$ goat serum. Cells were incubated in primary antibodies overnight.

The used primary antibodies were mouse anti-nestin monoclonal antibody (abcam; 1:500) mouse anti-MAP-2 monoclonal antibody (abcam; 1:300), and $\beta$-tubulin monoclonal antibody (abcam; 1:200). After washing, the cells were washed and incubated with the secondary antibody, fluorescein isothiocyanate (FITC; 1:1000) for one hour. Cells were mounted with 4,6-diamidino2-phenylindole (DAPI/PBS; 1:1000). Images were captured with an Olympus phase.

\section{Statistical analysis}

SPSS 13.0 software One-way analysis of variance (ANOVA) was used for analyzing the data, and then by Tukey post hoc test ( $p<0.05$ was considered significant).

\section{Results}

\section{Specific characteristics of hDPSCs}

The hDPSCs were attached, proliferated rapidly and filled the bottom of culture dish. They appeared as spindle shaped or with fibroblastic morphology (Fig. 1). As we showed in previous research, the mesenchymal markers, namely CD 44, CD 90, and CD 105, were detected via flow cytometry technique (Moayeri et al, 2017). Multilineage differentiation of hDPSCs was proved via confirming the osteogenic and adipogenic differentiation (Moayeri et al, 2017).

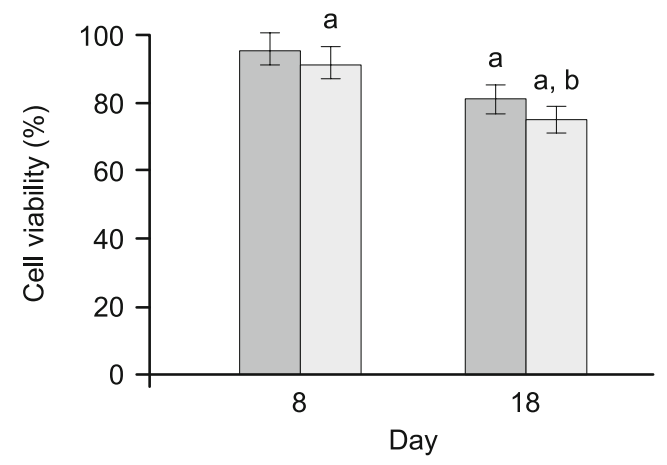

Fig. 5. MTT dates showing viability rates on days 8 and 18 of induction protocol. Significant decrease in control group on day 8 (a). Significant decrease in control group on day 18 (b).

\section{Derivation of NSCs from $h D P S C s$}

Following the fourth passage, hDPSCs were trypsinized, and cultured in non-adherence flasks containing neural stem cells medium supplemented with bFGF and EGF. Neurospheres were formed after 7-10 days (Fig. 2). The derivation of NSCs from hDPSCs was accompanied with a wide range of morphological changes.

\section{Derivation of mature neuron from NSCs}

NSCs transformed to mature neurons with branched morphology after induction protocol (Fig. 3).

Immunostaining data proved the expression of neuroepithelial markers including nestin in NSCs (Fig. 2). The differentiated mature neurons were immunopositive to specific markers as e.g. MAP-2 and $\beta$-tubulin (Fig. 4).

\section{Viability of hDPSCs during induction steps}

The survival rate of cells at the first stage of induction for experiment and control were evaluated via MTT test that showed $91.58 \pm 0.33 \%$ and $95.72 \pm 0.36 \%$, respectively. Also, the viability rate showed a significant decrease between case and control groups at the end of protocol $(\mathrm{p}<0.05)$ (Fig. 5). 


\section{Discussion}

Mesenchymal stem cells have an ability to differentiate into a neural phenotype after being exposed to neural inducers (Delaviz et al, 2014, Ghasemi et al, 2014, Le Blanc et al, 2005, Nazm Bojnordi et al, 2015). The hDPSCs arise from the neural crest and possibly are considered to be an appropriate cell source for neuronal generation usable in the treatment of neurodegenerative diseases (Yalvac et.al.2009, Huang et.al.2008).

Recent research showed that mesenchymal stem cells derived from bone marrow can convert into NSCs (Sanchez-Ramos et al, 2000, Ping et al, 2015, Darabi et al, 2013). We have used these protocols for hDPSCs which represent a promising therapeutic approach in neuroregenerative medicine. By using MSCs derived from human teeth, we have proved that under specific culture conditions, hDPSCs have the ability to form NSCs . Theses NSCs can differentiate into neurons and exhibit mature neural markers detectable via immunocytochemistry technique.

The potential of hDPSCs to differentiate into neurons has already been proven in previous studies, but the maturity of neurons has not yet been evaluated (Chang et al, 2014, Chun et al, 2016, Ghasemi et al, 2017). Therefore, we have designed an improved modification of induction technique for in vitro differentiation of hDPSCs into mature neurons.

NSCs-derived hDPSCs were transformed from the proliferation stage into the differentiation phase, they exhibited the morphology of neuroprogenitor cells and were immunopositive to nestin and NF68 markers. Our data are similar to those in previous reports in the sense that the neural differentiation of mesenchymal stem cells is associated with nestin and NF68 as neuroprogenitor markers (Haratizadeh et al, 2016).

Culturing the cells in neural stem cells medium supplemented with bFGF and EGF completed the neural differentiation and exhibited specific neural markers, namely MAP-2 and $\beta$-tubulin.

The generation of neural phenotype from hDPSCs which has been proved in this research is the basis for a proposition that hDPSCs are an appropriate alternative source for improving the treatment of neural damage in neuroregenerative medicine. Nevertheless, more investigation is needed to approve the functionality of mature neuron derived from hDPSCs.

\section{References}

1. Gronthos S, Brahim J, Li W, Fisher LW, Cherman N, Boyde A et al. Stem cell properties of human dental pulp stem cells. J Dent Res 2002; 81 (8): 531-535.

2. Sloan AJ, Smith AJ. Stem cells and the dental pulp: potential roles in dentine regeneration and repair. Oral Dis 2007; 13: 151-157.

3. Gronthos S, Mankani M, Brahim J, Robey PG, Shi S. Postnatal human dental -pulp stem cells (DPSCs) in vitro and invivo. Proc Nat Acad Sci 2000; 97 (25): 13625-13630.

4. Fu L, Zhu L, Huang Y, Lee TD, Forman SJ, Shih CC. Derivation of neural stem cells from mesenchymal stem cells: evidence for a bipotential stem cell population. Stem cells Dev 2008; 17: 1109-1122.
5. Moayeri A, Bojnordi MN, Haratizadeh S, Esmaeilnejad-Moghadam A, Alizadeh R, Hamidabadi HG. Transdifferentiation of human dental pulp stem cells into oligoprogenitor cells. Basic Clin Neurosci 2017; 8 (5): 387.

6. Le Blanc K, Pittenger M. Mesenchymal stem cells: progress toward promise. Cytotherapy 2005; 7: 36-45.

7. NazmBojnordi M, Ghasemi HH, Akbari E. Remyelination after lysophosphatidyl choline-induced demyelination is stimulated by bone marrow stromal cell-derived oligoprogenitor cell transplantation. Cells Tissues Organs 2015; 200 (5): 300-306.

8. Yalvac ME, Rizvanov AA et al. Potential role of dental stem cells in the cellular therapy of cerebral ischemia. Curr Pharm Des 2009; 15: 3908-3916.

9. Huang AHC, Chen YK et al. Isolation and characterization of dental pulp stem cells from a supernumerary tooth. J Oral Pathol Med 2008; 37: 571-574.

10. Sanchez-Ramos J, Song S, Cardozo-Pelaez F, Hazzi C, Stedeford T, Willing A, Freeman TB, Saporta S, Janssen W, Patel N, Cooper DR, Sanberg PR. Adult bone marrow stromal cells differentiate into neural cells in vitro. Exp Neurol 2000; 164: 247-256.

11. Gu P, Qiu FC, Han R, Zhang ZX, Dong C, Zhang LN, Wang YY, Ma QY, Yan BY. Efficient differentiation of neural stem cells induced by the rat bone marrow stromal cells. Int J Clin Exp Med 2015; 8 (5): 6713-6724.

12. Darabi S, Tiraihi T, Ruintan A, Abbaszadeh HA, Delshad A, Taheri T. Polarized neural stem cells derived from adult bone marrow stromal cells develop a rosette-like structure. In Vitro Cell Dev Biol Ani-m. 2013 Sep; 49 (8): 638-652.

13. Chang CC, Chang KC, Tsai SJ, Chang HH, Lin CP. Neurogenic differentiation of dental pulp stem cells to neuron-like cells in dopaminergic and motor neuronal inductive media. J Formos Med Assoc 2014; 113 (12): 956-965.

14. Chun SY, Soker S, Jang YJ, Kwon TG, Yoo ES. Differentiation of Human Dental Pulp Stem Cells into Dopaminergic Neuron-like Cells in Vitro. J Korean Med Sci 2016; 31 (2): 171-177.

15. Ghasemi Hamidabadi H, Rezvani Z, Nazm Bojnordi M, Shirinzadeh H, Seifalian A, Joghataei MT, Razaghpour M et al. Chitosan-intercalated Montmorillonite/Poly (Vinyl alcohol) Nanofibers as a Platform to Guide Neuron-like Differentiation of Dental Pulp Stem Cells. ACS Applied Materials Interfaces 2017; 9 (13): 11392-11404.

16. Haratizadeh S, Nazm Bojnordi M, Darabi S, Karimi N, Naghikhani M, Ghasemi Hamidabadi H, Seifi M. Condition medium of cerebrospinal fluid and retinoic acid induces the transdifferentiation of human dental pulp stem cells into neuroglia and neural like cells. Anat Cell Biol 2017; 50 (2): $107-114$.

17. Hermann A, Gastl R, Liebau S, Oana Popa M, Fiedler J, Boehm BO, Maisel M, Lerche H, Schwarz J, Brenner R, Storch A. Efficient generation of neural stem cell-like cells from adult human bone marrow stromal cells. J Cell Sci 2004 117: 4411-4422.

18. Ghasemi N, Razavi S, Mardani M, Esfandiari E, Salehi H, Zarkesh Esfahani SH. Transplantation of human adipose-derived stem cells enhances remyelination in lysolecithin-induced focal demyelination of rat spinal cord. Mol Biotechnol 2014; 56 (5): 470-478.

19. Delaviz H, Joghataie MT, Mehdizadeh M, Bakhtiyari M, Nobakht M, Khoei S. Transplantation of olfactory mucosa improve functional recovery and axonal regeneration following sciatic nerve repair in rats. Iran Biomed J 2008; 12 (4): 197-202.

20. Haratizadeh S, Bojnordi MN, Niapour A, Bakhtiari M, Hamidabadi HG. Improvement of neuroglial differentiation from human dental pulp stem cells using CSF. J Mazandaran Univ Med Sci 2016; 26 (140): 1-14.

Received April 28, 2018. Accepted May 29, 2018. 